\title{
Uso do Tramadol em Cães: uma Breve Revisão
}

\author{
Use of Tramadol in Dogs: a Brief Review
}

\author{
Lorraine Gabriela Trettene*a; Michele Lunardib; Isabelle Cristina Barros ${ }^{\mathrm{b}}$; Daniella Aparecida Godoi Kemper ${ }^{\mathrm{a}}$
}

${ }^{a}$ Unopar, Programa de Pós-Graduação Stricto Sensu em Saúde e Produção Animal. PR, Brasil.

bUniversidade de Cuiabá, Programa de Pós-Graduação Stricto Sensu em Biociência Animal. MT, Brasil.

*E-mail: lorrainetrettene@hotmail.com

\begin{abstract}
Resumo
O uso do tramadol vem sendo comumente e amplamente realizado na Medicina Veterinária nos últimos anos, o qual vem sendo utilizado tanto para dor aguda quanto para dor crônica. O tramadol é um opioide atípico com baixa afinidade por receptores $\mu$ (mu), e com ação monoaminérgica através da inibição da recaptação de norepinefrina e liberação de serotonina. O principal metabólito que é resultante da metabolização do tramadol nas isoenzimas do complexo P450 no fígado, é chamado de O-dismetiltramadol (M1), sendo o principal metabólito responsável pelo efeito analgésico do tramadol. No entanto, esse metabólito é encontrado em uma menor concentração em cães, quando comparado a gatos e humanos. E isso levou a um certo questionamento quanto a eficácia analgésica do tramadol em cães. Ademais, vários estudos já foram realizados avaliando os efeitos do tramadol, tanto em questões farmacocinéticas e farmacodinâmicas quanto em questões de avaliação de doses, vias de administração, e eficácia analgésica. Portanto, estudos a respeito do uso do tramadol em cães são crescentes, sendo necessário que os médicos veterinários se mantenham atualizados, para que possam empregar o fármaco de maneira eficaz. Diante de tais fatos, o presente trabalho teve como objetivo realizar uma revisão de literatura científica dos principais estudos relacionados ao uso do tramadol em cães.
\end{abstract}

Palavras-chave: Analgesia. O-desmetiltramadol. Opióide. Tramadol.

\begin{abstract}
The use of tramadol has been commonly and widely used in Veterinary Medicine in recent years, which has been used for both acute and chronic pain. Tramadol is an atypical opioid with low affinity for $\mu$ receptors, and with monoaminergic action through inhibition of norepinephrine reuptake and serotonin release. The main metabolite that results from the metabolization of tramadol in the isoenzymes of the P450 complex in the liver, is called O-dismethyltramadol (M1), being the main metabolite responsible for the analgesic effect of tramadol. However, this metabolite is found in a lower concentration in dogs, when compared to cats and humans. This led to some questioning as to the analgesic efficacy of tramadol in dogs. In addition, several studies have been carried out evaluating the effects of tramadol, both on pharmacokinetic and pharmacodynamic issues and on questions of dose assessment, routes of administration, and analgesic efficacy. Therefore, studies on the use of tramadol in dogs are growing, and it is necessary that veterinarians keep up to date, so that they can use the drug effectively. Given these facts, the present study aimed to conduct a scientific literature review of the main studies related to the use of tramadol in dogs.
\end{abstract}

Keywords: Analgesia. O-desmethyltramadol. Opioid. Tramadol.

\section{Introdução}

Os opióides são os mais antigos e mais potentes fármacos analgésicos conhecidos, sendo os principais agentes utilizados para o controle da dor pós-operatória de ovariohisterectomia em cadelas (MWANGI et al., 2018). Os efeitos dos opioides são mediados pela afinidade que este possui pelo receptor específico, assim, os fármacos com afinidade a diferentes receptores produzem vários efeitos clínicos, portanto, os opioides mimetizam os efeitos decorrentes da ligação dos opioides endógenos. Os receptores opiáceos conhecidos são o mu $(\mu)$, kappa $(\kappa)$, sigma $(\sigma)$ e delta $(\delta)$ (FANTONI; MASTROCINQUE, 2010).

O tramadol é um opioide atípico, sendo um analgésico de ação central que possui fracas propriedades agonistas opioides e ação sobre a recaptação de norepinefrina e serotonina. Quando o tramadol é metabolizado, ele produz um metabólito chamado O-dismetiltramadol (M1) resultante de seu metabolismo pelas isoenzimas do complexo P450 no fígado e esse é o principal metabólito conhecido que promove efeito analgésico (GROND; SABLOTZKI, 2004). Os cães apresentam menor concentração do metabólito M1 do que humanos e gatos, o que levou ao questionamento sobre sua eficácia na espécie. Contudo, alguns estudos clínicos demonstraram analgesia suficiente para o tratamento da dor moderada em cães (MASTROCINQUE; FANTONI, 2003; CARDOZO et al., 2014; KAKA et al., 2018).

Devido a essa menor concentração do principal metabólito conhecido do tramadol que promove efeito analgésico ser observado em menor concentração em cães, muitos veterinários têm receio em utilizar o fármaco. Portanto, o objetivo deste trabalho é apresentar uma revisão das evidências atuais de estudos experimentais e clínicos envolvendo o tramadol. 


\section{Desenvolvimento}

\subsection{Metodologia}

Trata-se de uma revisão bibliográfica onde as buscas foram realizadas no período de 07 de maio de 2020 à 12 de junho de 2020 em bases de dados científicos, predominantemente, utilizadas no âmbito institucional: Google Acadêmico, SciELO, SCOPUS, Pubmed/Medline. Foram utilizados os seguintes descritores: "pain", "tramadol”, “dog”, "pharmacokinetics".

Para composição dessa revisão, foram escolhidos os seguintes critérios de inclusão: estudos relacionados à farmacocinética e/ou á farmacodinâmica do tramadol em cães; estudos relacionados à eficácia analgésica do tramadol em cães; artigos disponíveis na integra; artigos publicados entre os anos 2000 e 2020. Foram encontrados 12.317 artigos, dos quais 31 foram selecionados, sendo que estes se enquadravam nos critérios de inclusão, além de a maioria apresentarem data de publicação mais recente entre o período estabelecido. Os critérios de exclusão foram: todos os artigos cujo estudos não estavam relacionados à farmacocinética e ou/farmacodinâmica do tramadol em cães, ou à eficácia analgésica do tramadol em cães; artigos que não compreendiam o período entre os anos 2000 e 2020; e artigos que não estivessem disponíveis na integra. Os artigos que foram excluídos apresentavam um ou mais critérios de exclusão e/ou apresentavam informações repetidas que foram encontradas em artigos mais recentes.

\subsection{Discussão}

\subsubsection{Metabolismo do tramadol em cães}

O tramadol é um opioide comumente utilizado para controlar condições dolorosas de origem aguda ou crônica de intensidade leve à moderada em cães. Contudo, seu uso ainda é controverso, pois o principal metabólito responsável pela analgesia do tramadol, o metabólito ativo O-desmetiltramadol (M1), resultante de seu metabolismo pelas enzimas do complexo P450 no fígado, é encontrado em menor concentração em cães quando comparado à gatos e humanos, o que levou ao questionamento de sua eficácia nos cães. (GIORGI et al., 2010; KUKANICH; PAPICH, 2011; BENITEZ et al., 2015).

Ademais, há estudos que indicam alta variabilidade na resposta entre cães (KONGARA et al., 2013; CARDOZO et al., 2014 ; DELGADO et al., 2014 ; KOGEL et al., 2014) que pode ser devido á metabolização variável do tramadol pelas enzimas do citocromo P450 (CYP), sendo por consequência de diferenças genéticas, interações medicamentosas ou outras influências extrínsecas (MARTINEZ et al., 2019).

Jimenez et al. (2016), realizaram um estudo para identificar os P450s responsáveis pela formação de M1 e M2 no fígado canino, e foi revelado que o M1 (O-desmetiltramadol) é formado exclusivamente pelo CYP2D15 canino, enquanto M2 (N-desmetiltramadol) é formado por vários CYPs, incluindo
CYP2B11, CYP3A12, CYP2C21 e CYP2C41. E isso é semelhante ao metabolismo de medicamentos no humano, pois o M1 é formado apenas pelo CYP2D6, enquanto o M2 é formado pelo CYP2B6 e CYP3A4. Nesse mesmo estudo realizado por Jimenez et al. (2016), foi revelado que os microssomas hepáticos de cães (DLMs) quando comparado ao de gatos e humanos, não produzem altas concentrações do metabólito M1 em relação ao metabólito M2. E isso pode explicar as concentrações relativamente baixas de M1 em cães (GIORGI et al., 2009a; GIORGI et al., 2009b; KUKANICH; PAPICH, 2011; ITAMI et al., 2013; KOGEL et al., 2014) comparado com gatos (PYPENDOP; ILKIW, 2008; PYPENDOP et al., 2009; CAGNARDI et al., 2011) e seres humanos (SCOTT; PERRY, 2000; ARDAKANI; ROUINI, 2007; QUETGLAS et al., 2007). E isso é de extrema importância clínica, pois acredita-se que M1 tenha propriedades analgésicas mediadas pela atividade agonista $\mu$ em doses terapêuticas (GILLEN et al., 2000).

Além da metabolização do tramadol em M1, o tramadol é metabolizado extensivamente em N-desmetiltramadol (M2). E, os metabólitos M1 e M2 também são metabolizados em N, O-didesmetiltramadol (M5), que é o principal metabólito encontrado no plasma e na urina de cães que recebem tramadol (KUKANICH; PAPICH, 2011). Após a administração de tramadol em cães, o M5 foi encontrado no plasma em concentrações similares às concentrações de M2, mas, em concentrações mais altas (cerca de 20 vezes) que as concentrações de M1 (GIORGI et al., 2009a; GIORGI et al., 2009b; KUKANICH; PAPICH, 2011). Contudo, o M5 tem eficácia e potência do agonista $\mu$ muito mais fracos (cerca de 30 vezes) quando comparado ao M1 em testes in vitro (GILLEN et al., 2000).

Um estudo em ratos indicou que M5 tem baixa penetração no sistema nervoso central (SNC), onde as taxas de concentração no líquido cefalorraquidiano após a administração de tramadol foram inferiores a 0,1 para M5, enquanto M1, M2 apresentaram proporções de 0,3 e 0,4 respectivamente (SHEIKHOLESLAMI et al., 2016). Essa baixa concentração de M5 (3 vezes menor que M1) pode ser uma consequência de uma maior polaridade e menor solubilidade da membrana, ou efluxo ativo do SNC por um transportador de membrana, como a glicoproteína-p. Devido a fraca permeabilidade cerebral e a baixa potência e eficácia de M5, a formação de M5 a partir de M1 pode contribuir para a redução dos efeitos antinociceptivos $\mu$ de M1. Em cães, o principal CYP responsável pela formação de M5 a partir de M1 é o CYP2C21, e o CYP2D15 é exclusivamente responsável pela formação de M5 a partir de M2 (JIMENEZ et al., 2018).

Além disso, um recente estudo indicou que a coadministração oral de tramadol com fluconazol aumenta acentuadamente as concentrações plasmáticas e urinárias de tramadol e o metabolito de O-desmetiltramadol em cães 
saudáveis. Neste estudo, realizado por Jimenez et al. (2019), a concentração média de M1 no plasma após 4 horas da coadministração de tramadol com fluconazol foi de $54 \mathrm{ng} /$ $\mathrm{ml}$, o que é superior ao alcançado em todos os estudos anteriores que avaliaram efeitos do tramadol sobre os limiares nociceptivos (KUKANICH; PAPICH, 2011; KOGEL et al., 2014; SCHUTTER et al., 2017); contudo, essa concentração plasmática ainda é um pouco menor do que a concentração plasmática eficaz média de $\mathrm{M} 1 \mathrm{de} 113 \mathrm{ng} / \mathrm{ml}$, a qual foi relatada para gatos usando um método de teste de limiar térmico (PYPENDOP et al., 2009).

Entretanto, a ação antinociceptiva do tramadol no cão ainda não é totalmente compreendida e pode envolver efeitos sobre os adrenoceptores $\alpha 2$, bem como a inibição da recaptação da norepinefrina, além da ação agonista em receptor $\mu$. E, apesar das baixas concentrações plasmáticas de tramadol e seu metabólito ativo, a eficácia analgésica já foi relatada experimentalmente e clinicamente (MASTROCINQUE; FANTONI, 2003; KUKANICH; PAPICH, 2011; MALEK et al., 2012; CARDOZO et al., 2014; KAKA et al., 2018).

\subsubsection{Analgesia do tramadol em cães}

Em relação a eficácia clínica da analgesia do tramadol em cães, muitas dúvidas surgem, isso devido à baixa produção do metabólito O-dismetiltramadol (M1) nos cães, assunto o qual já foi discutido no tópico anterior. No entanto, inúmeros estudos já relataram a eficácia analgésica do tramadol em cães.

Há trabalhos na literatura que demonstram que o tramadol apresenta eficácia analgésica em cães, como um estudo realizado por Kaka et al. (2018), no qual as cadelas receberam tramadol, na dose de $4 \mathrm{mg} / \mathrm{kg}$ por via intravenosa (IV), como medicação pré-anestésica em ovariohisterectomia, e não houve necessidade de resgate analgésico em nenhum dos 12 animais envolvidos no estudo, evidenciando a eficácia analgésica do fármaco em ovariohisterectomia em cadelas. Outro estudo também relatou a eficácia analgésica do tramadol, onde foi administrado $2 \mathrm{mg} / \mathrm{kg} / \mathrm{IV}$ após a indução da anestesia em cadelas que foram submetidas à ovariohisterectomia, e foi revelado que o tramadol pode ser administrado com segurança em cadelas para controlar a dor no pós-operatório de ovariohisterectomia (MASTROCINQUE; FANTONI, 2003).

Em um recente estudo, a administração de tramadol intranasal na dose de $4 \mathrm{mg} / \mathrm{kg}$ pareceu ser tão eficaz quanto os tratamentos intravenosos de tramadol e metadona no tratamento da dor de cães após ovariohisterectomia eletiva (DI SALVO et al., 2020).

Contudo, Cardozo et al. (2014), em um estudo onde foi realizada a avaliação da dor pós-operatória de cirurgias ortopédicas, observou-se que o tramadol na dose de $4 \mathrm{mg} / \mathrm{kg} /$ IM administrado na MPA, teve menor efeito analgésico em comparação com a metadona. Entretanto, quando os animais foram resgatados com o acréscimo da dose do tramadol de 1 $\mathrm{mg} / \mathrm{kg}$, houve analgesia satisfatória. Portanto, de acordo com os resultados de Cardozo et al. (2014) podemos sugerir que ajustes individuais na dosagem podem ser necessários para promover analgesia adequada e levando-se em consideração também o tipo de estímulo doloroso.

Em contrapartida, também há estudos que relatam que o tramadol não foi tão eficaz para analgesia, como em um estudo onde o tramadol foi comparado ao firocoxibe no pós-operatório de cirurgia ortopédica, o tramadol não promoveu eficácia analgésica satisfatória e foi inferior ao firocoxibe, porém, devemos levar em consideração que a via de administração foi oral (4 a $5 \mathrm{mg} / \mathrm{kg}$ ) e os fármacos foram administrados de forma preemptiva (DAVILA et al., 2013). Da mesma forma, em cirurgia de enucleação em cães, o carprofeno promoveu analgesia superior ao tramadol, porém ambos também foram administrados por via oral (DELGADO et al., 2014). Segundo Kukanich e Papich (2004), a administração oral de tramadol pode sofrer uma variação individual considerável nos níveis séricos do fármaco nos cães.

Assim, também podemos enfatizar os efeitos de alta variação interindividual e diferenças associadas à raça de cães na expressão do citocromo P450 no metabolismo de medicamento, sendo de extrema importância para vermos a variabilidade da disposição de medicamentos entre uma população canina geneticamente diversa (MARTINEZ et al., 2019).

\section{Conclusão}

Foi demonstrado que o tramadol pode ser eficaz e proporcionar analgesia em cães, no entanto, devemos levar em consideração o estímulo doloroso, ajustes individuais na dosagem do fármaco, e a variação individual considerável nos níveis séricos do fármaco nos cães.

Portanto, podemos concluir que ainda são necessários mais estudos na área de farmacologia para avaliar o principal mecanismo de ação responsável pela analgesia conferida pelo tramadol em cães.

\section{Referências}

ARDAKANI, Y.H.; ROUINI, M.R. Pharmacokinetics of tramadol and its three main metabolites in healthy male and female volunteers. Biopharm. Drug Disposition, v.28, n.9, p.527534, 2007. doi: 10.1002/(ISSN)1099-081X.

BENITEZ, M.E. et al. Pharmacokinetics of hydrocodone and tramadol administered for control of postoperative pain in dogs following tibial plateau leveling osteotomy. Am. J. Vet. Res., v.76, n.9, p.763-70, 2015. doi: 10.2460/ajvr.76.9.763.

CAGNARDI, P. et al. Pharmacokinetics, intraoperative effect and postoperative analgesia of tramadol in cats. Res. Vet. Scie., v.90, n.3, p.503-509, 2011. doi: 10.1016/j.rvsc.2010.07.015.

CARDOZO, L. et al. Evaluation of the effects of methadone and tramadol on postoperative analgesia and serum interleukin-6 in dogs undergoing orthopaedic surgery. BMC Vet. Res., v.10, n.194, p.1-7, 2014. doi: 10.1186/s12917-014-0194-7.

DAVILA, D. et al. Comparison of the analgesic efficacy of perioperative firocoxib and tramadol administration in dogs undergoing tibial plateau leveling osteotomy. $\mathrm{J}$. Am. Vet. Med. Assoc., v.243, n.2, p.225-231, 2013. doi: 10.2460/ 
javma.243.2.225.

DELGADO, C. et al. Comparison of carprofen and tramadol for postoperative analgesia in dogs undergoing enucleation. $\mathrm{J}$. Am. Vet. Med. Assoc., v.245, n.12, p.1375-1381, 2014. doi: 10.2460/ javma.245.12.1375.

DI SALVO, A. et al. Pharmacokinetics and analgesic efficacy of intranasal administration of tramadol in dogs after ovariohysterectomy. Vet. Anaesthesia Analgesia, v. 47, n.4, p. 557-566, 2020. doi: 10.1016/j.vaa.2019.12.011.

FANTONI, D.T.; MASTROCINQUE, S. Fisiopatologia e controle da dor aguda. In: FANTONI, D.T.; CORTOPASSI, S.R.G. Anestesia em cães e gatos. São Paulo: Roca, 2010. p.521541.

GIORGI, M. et al. Pharmacokinetic and urine profile of tramadol and its major metabolites following oral immediate release capsules administration in dogs. Vet. Res. Communic., v.33, n.8, 875-885, 2009a. doi: 10.1007/s11259-009-9236-1.

GIORGI, M. et al. Pharmacokinetic evaluation of tramadol and its major metabolites after single oral sustained tablet administration in the dog: a pilot study. Vet. J., v.180, n.2, p.253-255, 2009b. doi: 10.1016/j.tvj1.2007.12.011

GIORGI, M. et al. Pharmacokinetics of tramadol and metabolites after injective administrations in dogs. Pol. J. Vet. Sci., v.13, n.4, p.639-644, 2010. doi: 10.2478/v10181-010-0027-y.

GILLEN, C. et al. Affinity, potency and efficacy of tramadol and its metabolites at the cloned human $\mu$-opioid receptor. NaunynSchmiedeberg's Arch. Pharm., v.362, n.2, p.116-121, 2000. doi: $10.1007 / \mathrm{s} 002100000266$

GROND, S.; SABLOTZKI, A. Clinical pharmacology of tramadol. Clin. Pharm., v.43, n.13, p.879-923, 2004. doi: 10.2165/00003088-200443130-00004.

ITAMI, T. et al. Effects of a single bolus intravenous dose of tramadol on minimum alveolar concentration (MAC) of sevoflurane in dogs. J. Vet. Med. Scie., v.75, n.5, p.613-618, 2013. doi: 10.1292/jvms.12-0208.

JIMENEZ, T.E.P. et al. Tramadol metabolism to O-desmethyl tramadol (M1) and N-desmethyl tramadol (M2) by dog liver microsomes: Species comparison and identification of responsible canine cytochrome P-450s (CYPs). Drug Metabol. Disp., v.44, n.12, p.1963-1972, 2016. doi: 10.1124/dmd.116.071902.

JIMENEZ, T.E.P. et al. Identification of canine cytochrome P-450s (CYPs) metabolizing the tramadol $(+)-\mathrm{M} 1$ and $(+)-$ M2 metabolites to the tramadol (+)-M5 metabolite in dog liver microsomes. Pharmacol. Ther., v.41, n.6, p.815-824, 2018. doi: 10.1111/jvp.12706.

JIMENEZ, T.E.P. et al. Oral Coadministration of Fluconazole with tramadol markedly increases plasma and urine concentrations of tramadol and the o-desmethyltramadol metabolite in healthy dogs. Drug Metabol. Disp., v.47, n.1, p.15-25, 2019. doi: 10.1124/ dmd.118.083444.

KAKA, U. et al. Pre-emptive multimodal analgesia with tramadol and ketamine-lidocaine infusion for suppression of central sensitization in a dog model of ovariohysterectomy. J. Pain Res., v.11, p.743-752, 2018. doi: 10.2147/JPR.S152475.

KOGEL, B.; TERLINDEN, R.; SCHNEIDER, J. Characterisation of tramadol, morphine and tapentadol in an acute pain model in Beagle dogs. Vet. Anaest. Anal., v.41, n.3, p.297-304, 2014. doi: 10.1111/vaa.12140.

KONGARA, K. et al. Effects of tramadol or morphine in dogs undergoing castration on intra-operative electroencephalogram responses and post-operative pain. New Zealand Vet. J., v.61, n.6, p.349-353, 2013. doi: 10.1080/00480169.2013.780280.

KUKANICH, B.; PAPICH, M.G. Pharmacokinetics of tramadol and the metabolite O-desmethyltramadol in dogs. J. Vet. Pharmacol. Ther, v.27, n.4, p.239-46, 2004. doi: 10.1111/j.13652885.2004.00578.x.

KUKANICH, B.; PAPICH, M.G. Pharmacokinetics and antinociceptive effects of oral tramadol hydrochloride administration in Greyhounds. Am. J. Vet. Res., v.72, n.2, p.256262, 2011. doi: 10.2460/ajvr.72.2.256.

MASTROCINQUE, S.; FANTONI, D.T. Comparison of preoperative tramadol and morphine for the control of early postoperative pain in canine ovariohysterectomy. Vet. Anaesth. Analg., v.30, p.220-228, 2003. doi: 10.1046/j.14672995.2003.00090.x.

MALEK, S. et al. Effect of analgesic therapy on clinical outcome measures in a randomized controlled trial using client-owned dogs with hip osteoarthritis. BMC. Vet. Res., v.185, n.8, p.1-17, 2012. doi: 10.1186/1746-6148-8-185.

MARTINEZ, S.E. et al. Absolute quantitation of drugmetabolizing cytochrome p450 enzymes and accessory proteins in dog liver microsomes using label-free standard-free analysis reveals interbreed variability. Drug Metabol. Disposition, v.47, n.11, p.1314-1324, 2019. doi: 10.1124/dmd.119.088070.

MWANGI, W.E. et al. A systematic review of analgesia practices in dogs undergoing ovariohysterectomy. Vet. World, v.11, n.12, p.1725-1735, 2018. doi: 10.14202/vetworld.2018.1725-1735.

PYPENDOP, B. H.; ILKIW, J.E. Pharmacokinetics of tramadol, and its metabolite O-desmethyl-tramadol, in cats. J. Vet. Pharmacol. Ther, v.31, n.1, p.52-59, 2008. doi: 10.1111/j.13652885.2007.00921.x.

PYPENDOP, B. H.; SIAO, K.T.; ILKIW, J. E. Effects of tramadol hydrochloride on the thermal threshold in cats. Am. J. Vet. Res. v.70, n.12, p.1465-1470, 2009. doi: 10.2460/ ajvr.70.12.1465.

QUETGLAS, E.G. et al. Stereoselective pharmacokinetic analysis of tramadol and its main phase I metabolites in healthy subjects after intravenous and oral administration of racemic tramadol. Biopharm. Drug Disposition, v.28, n.1, p.19-33, 2007. doi: 10.1002/(ISSN)1099-081X.

SCHUTTER, A.F.; TUNSMEYER, J; KASTNER, S.B.R Influence of tramadol on acute thermal and mechanical cutaneous nociception in dogs. Vet. Anaesth. Analg., v.44, n.2, p.309-316, 2017. doi: 10.1016/j.vaa.2016.02.003.

SCOTT, L.J.; PERRY, C.M. Tramadol: A review of its use in perioperative pain. Drugs, v.60, n.1, p.139-176, 2000. doi: 10.2165/00003495-200060010-00008.

SHEIKHOLESLAMI, B. et al. Evaluation of the route dependency of the pharmacokinetics and neuro-pharmacokinetics of tramadol and its main metabolites in rats. Euro. J. Pharm. Scie., v.92, p.5563, 2016. doi: 10.1016/j.ejps.2016.06.021. 